\title{
Dynamic Surface Reconstruction of 2D Ruddlesden-Popper Halide Perovskite under e-Beam Irradiation
}

\author{
Hee Joon Jung ${ }^{1}$, Constantinos C. Stoumpos ${ }^{2}$, Mercouri G. Kanatzidis ${ }^{2,3}$ and Vinayak P. Dravid ${ }^{1}$ \\ ${ }^{1}$ Department of Materials Science and Engineering, Northwestern University, Evanston, Illinois 60208, \\ USA \\ ${ }^{2}$ Department of Chemistry, Northwestern University, Evanston, Illinois 60208, USA \\ ${ }^{3}$ Materials Science Division, Argonne National Laboratory, Argonne, IL 60439, USA
}

Two-dimensional (2D) Ruddlesden-Popper (RP) halide perovskites, $\mathrm{C}_{2} \mathrm{MA}_{n-1} \mathrm{~Pb}_{n} \mathrm{I}_{3 n+1} \quad(\mathrm{C}=$ bulky ammonium cation; $\mathrm{MA}=$ methyl-ammonium) with low $\mathrm{n}(\mathrm{n}<5)$, have been gathering an broad attention in solar cell or optoelectronic application due to not only long term stability under oxygen environment but also unique optoelectronic property such as bandgap tunability by thickness control of quantum well $(\mathrm{QW})$ halide perovskite and sharp absorption edges in the visible range against 3D halide perovskite $(\mathrm{n}=\infty)$ such as $\mathrm{MAPbI}_{3}$ [1-5], where $\mathrm{MA}$ is methyl-ammonium $\left(\mathrm{CH}_{3} \mathrm{NH}_{3}{ }^{+}\right) . \mathrm{A} \mathrm{BA}_{2} \mathrm{MA}_{2} \mathrm{~Pb}_{3} \mathrm{I}_{10}$ is one species ( $\mathrm{n}=3$ type) of the 2D-RP perovskites and has repeating sequence of three monolayers of semiconducting hybrid organic-inorganic $\mathrm{MA}_{2} \mathrm{~Pb}_{3} \mathrm{I}_{10}$ perovskites and $\mathrm{QW}$ barrier insulating molecules, which are butyl-ammonium molecules $\left(\mathrm{BA}, \mathrm{CH}_{3}\left(\mathrm{CH}_{2}\right)_{3} \mathrm{NH}_{3}{ }^{+}\right)$separating the semiconducting layers as can be seen in Fig. 1. While the electron beam irradiation effect on the 3D halide perovskite materials have been investigated [5-9], studies on 2D halide perovskites are lacking.

Herein we present dynamic surface modification on a $\mathrm{BA}_{2} \mathrm{MA}_{2} \mathrm{~Pb}_{3} \mathrm{I}_{10}(\mathrm{n}=3)$ 2D perovskite with $\mathrm{BA}$ (butyl-ammonium, $\mathrm{CH}_{3}\left(\mathrm{CH}_{2}\right)_{3} \mathrm{NH}_{3}+$ ) working as $\mathrm{QW}$ barrier molecules induced by electron beam in transmission electron microscope. We observed various surface dynamics in atomic level under electron irradiation such as lateral growth in c planes of polytypic $\mathrm{PbI}_{2}$ with $3 \mathrm{R}, 4 \mathrm{H}$ and $2 \mathrm{H}$ structures at the edge surface of the 2D perovskite accompanied with simultaneous annihilation of surface plane as can be seen in Fig. 1 and 2. Furthermore, we observe newly reconstructed regions generated by long electron irradiation which are confirmed to be $\mathrm{PbI}_{2}$, driven by local radiolysis due to internal energy increase of electron momentum transfer rather than knock-on damage mechanism. Moiré fringe from $\mathrm{PbI}_{2}$ formation on the $\mathrm{BA}_{2} \mathrm{MA}_{2} \mathrm{~Pb}_{3} \mathrm{I}_{10}$ is also observed. Direct observation on the atomically reconstructed surface of the 2D halide perovskite driven by increased internal energy will provide insight of stability under total dose level of e-beam irradiation in TEM.

[1] C. C. Stoumpos et al., Chem. Mater. 28, (2016), p. 2852-2867.

[2] C. C. Stoumpos et al., Chem 2, (2017), p. 427-440.

[3] D. H. Cao et al., J. Am. Chem. Soc. 137, (2015), p. 7843-7850.

[4] I. C. Smith et al., Angew. Chem. Int. Ed. 53, (2014), p. 11232-11235.

[5] H. Tsai et al., Nature 536, (2016), p. 312-316.

[6] Z. Dang et al., ACS Omega 2, (2017), p. 5660-5665.

[7] N. B. Yi et al., Adv. Mater. 29, (2017), p. 1701636.

[8] Z. Dang et al., ACS Nano 11, (2017), p. 2124-2132.

[9] Y. Tong et al., Angew. Chem. Int. Ed. 55, (2016), p. 13887. 
[10] This work made use of the EPIC facility of Northwestern University's NUANCE Center, which has received support from the Soft and Hybrid Nanotechnology Experimental (SHyNE) Resource (NSF ECCS-1542205); the MRSEC program (NSF DMR-1720139) at the Materials Research Center; the International Institute for Nanotechnology (IIN); the Keck Foundation; and the State of Illinois, through the IIN.

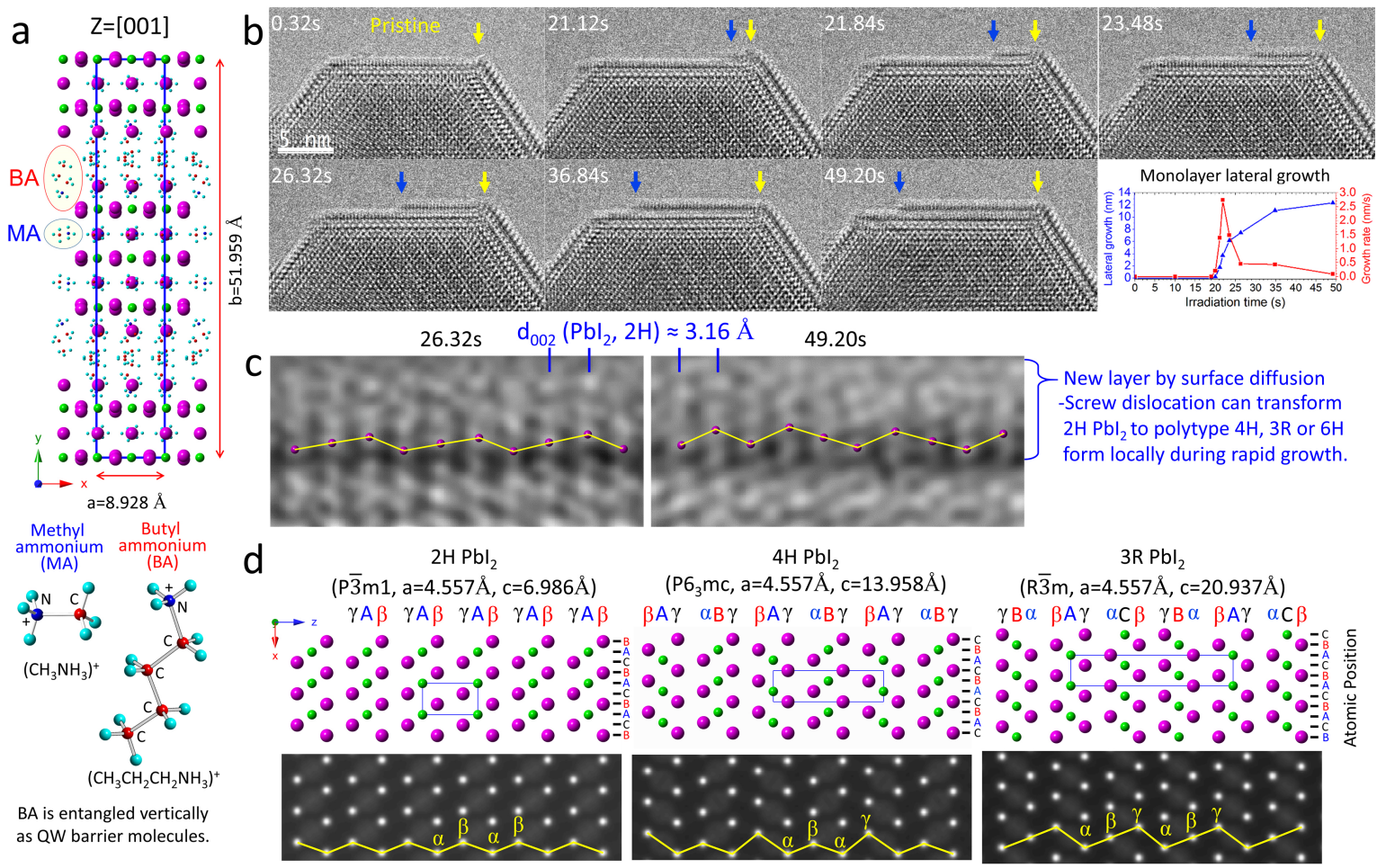

Figure 1. a. Cross-section projection along [001] with unit cell defined as blue line and organic molecule of MA and BA. b. Serial snapshots showing lateral growth of a new layer at the edge of $\mathrm{BA}_{2} \mathrm{MA}_{2} \mathrm{~Pb}_{3} \mathrm{I}_{10}$ and a graph displaying total lateral growth length (blue) and growth rate (red). c. HRTEMs at the edge of [414] zone axis of $\mathrm{BA}_{2} \mathrm{MA}_{2} \mathrm{~Pb}_{3} \mathrm{I}_{10}$ at 26.32s and 49.20s showing lateral growth of $\mathrm{PbI}_{2}$ (002) plane displaying different terminal configuration of iodine atoms at the interface. e. Atomic configurations (top) and the simulated images (bottoms) of $2 \mathrm{H}, 4 \mathrm{H}$ and $3 \mathrm{R} \mathrm{PbI} 2$.

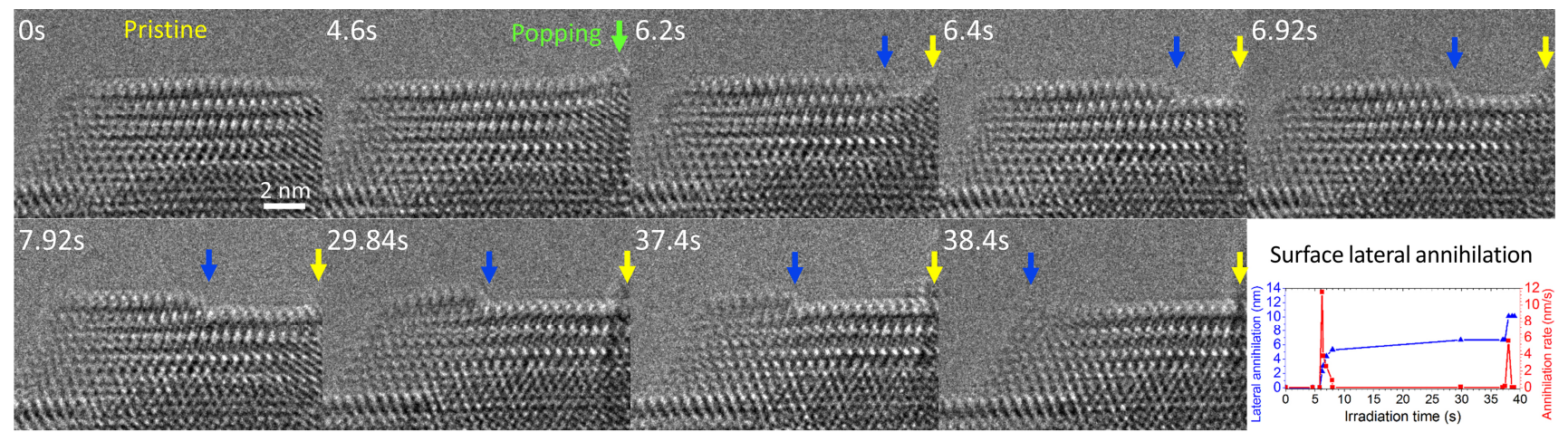

Figure 2. a. Snapshot series of later surface annihilation at the edge surface of the $\mathrm{BA}_{2} \mathrm{MA}_{2} \mathrm{~Pb}_{3} \mathrm{I}_{10}$ during electron irradiation of $38.4 \mathrm{sec}$ with total dose of $\sim 1.2 \times 10^{6} \mathrm{e} / \mathrm{nm}^{2}$ and a plot showing annihilation length (blue) and rate (red). 